\title{
Ultra Wideband Coplanar Waveguide Based Impedance Transformer using Slow-wave Electrodes
}

\author{
Xia Yao ${ }^{1}$ and Nicolas A. F. Jaeger \\ Department of Electrical and Computer Engineering \\ University of British Columbia \\ 2332 Main Mall, Vancouver, British Columbia, Canada V6T 1Z4
}

\begin{abstract}
This paper presents a new type of broadband impedance transformer loaded with capacitive fins (ITF) suitable for use in the frequency range $10 \mathrm{GHz}$ to $70 \mathrm{GHz}$. Compared with conventional, unloaded, tapered impedance transformers, these ITF structures extend the impedance matching range and the operating bandwidth for the same amount of on-chip realestate. We have designed ITFs capable of impedance matching resistive loads from $\sim 10 \Omega$ to $\sim 229 \Omega$, on a $650 \mu \mathrm{m}$ thick GaAs substrate over about a $60 \mathrm{GHz}$ bandwidth. Design examples are used to demonstrate the flexibility of these ITF structures.
\end{abstract}

Broadband impedance transformer, slow-wave electrode theory, capacitive loading fins, coplanar waveguide.

\section{Introduction}

The growing demand for channel transmission capacity in the telecommunications industry has lead to a perpetual interest in developing millimeter wave electronic devices. Impedance transformers are important components at millimeter wave frequencies and are typically used to reduce reflections between mismatched loads. This allows cleaner signal transfer and higher bit rates. In recent years, attractive impedance transformers based on microstrip and coupled transmission lines have been demonstrated ${ }^{1-3}$. These proposed impedance transformers would operate below $20 \mathrm{GHz}$ and are several centimeters long. Also, miniaturized impedance matching circuits utilizing meandered coplanar waveguide $(\mathrm{CPW})$ were demonstrated ${ }^{4,5}$ for MMIC wireless RF front-end applications. In these structures, the $\lambda / 4 \mathrm{CPW}$ transmission lines are folded together to achieve size reduction. However, the impedance matching range of these devices was not explored.

In this paper, a new type of impedance transformer loaded with capacitive fins (ITF) is proposed. Our ITF is based on tapered CPW impedance transformers (IT). Traditionally, there are many types of tapered microwave ITs, namely the triangularly tapered transformer, the exponentially tapered transformer, and the Klopfenstein tapered transformer ${ }^{6}$. Of these, the exponentially tapered transformer (ETT) offers low reflection coefficient, wide operating bandwidth, and ease of design. Though other substrate thicknesses are possible, our ITFs are designed on a $650 \mu \mathrm{m}$, conductor-backed GaAs substrate. The impedance values along the length of the ITF structures are matched to those of ETT. Capacitive loading fins are added to the center and ground electrodes to raise the effective microwave index which, in turn, increases the operating bandwidth and impedance matching range of the impedance transformer. We have used the high frequency EM analysis program SONNET ${ }^{\circledR}$ V.12 to design and analyze the ITF structures. In addition to the theory of operation, several design examples will be presented in this paper.

\footnotetext{
${ }^{1} \mathrm{X}$. Yao is currently at Imperial College Business School, Imperial College London, South Kensington Campus, London, United Kingdom SW7 2AZ; email: terry_jaxa@hotmail.com
}

Photonics North 2011, edited by Raman Kashyap, Michel Têtu, Rafael N. Kleiman, Proc. of SPIE Vol. 8007, 80070G · C 2011 SPIE · CCC code: 0277-786X/11/\$18 - doi: 10.1117/12.905674 


\section{Tapered CPW Impedance Transformer}

The CPW must be carefully designed to eliminate any undesired propagation modes. For the frequencies and dimensions considered here, there are, typically, four possible propagation modes for a CPW line, namely the slotline, the microstrip, the coplanar, and the surface wave parallel plate modes ${ }^{7}$. In our designs/simulations, the slotline mode is not present. Also, given that $\mathrm{H}$ is $650 \mu \mathrm{m}$, we chose values of $\mathrm{W}$ and $\mathrm{G}$ so as to suppress the surface wave parallel plate mode, see Figure 1. Additionally, Wg is the same for both outer, ground electrodes and is chosen to be $200 \mu \mathrm{m}$. This ensures that the potential on the ground conductor approaches zero when the center conductor is excited ${ }^{7}$. As a result, our CPW guides only a single coplanar mode.

Once the dimensions $\mathrm{W}, \mathrm{G}$, and $\mathrm{Wg}$ are decided upon, impedance matching can be achieved by tapering the center electrode ${ }^{6}$. ETTs exhibit high pass characteristics, where $\mathbf{S} 21$ first rises with respect to frequency, reaches a maximum at the cutoff frequency and then tapers off as the frequency further increases subject to microwave loss. In impedance transformers the term "cutoff frequency" is used for the frequency at which the first null of the reflection coefficient occurs $^{6}$ which is different from how the term "cut-off frequency" is often used to mean the $3 \mathrm{~dB}$ frequency. Using this definition, the cutoff frequency, $f_{\text {cutofff }}$, of ETT can be expressed as ${ }^{6}$

$$
f_{\text {cutoff }}=\frac{c}{2 L n_{\text {eff }}}
$$

where $c$ is the speed of light, $L$ is the length of the IT, and $n_{\text {eff }}$ is the effective microwave index. In order to achieve a wider operating bandwidth, the designer can increase $n_{\text {eff }}$ and/or $L$. $n_{\text {eff }}$ is fixed at $\sim 2.6$ for standard CPW on GaAs substrates so only $L$ can be changed. Given a minimum feature size of $1 \mu \mathrm{m}$, we have designed ETTs with impedance matching ranges from $\sim 24 \Omega$ to $\sim 229 \Omega$. In what follows, we show that we can use interdigitated capacitive loading fins in CPW to significantly increase $n_{\text {eff }}$ and, thereby, reduce the length of the impedance transformer, lower the minimum impedance that can be matched, and significantly lower the cutoff frequency (in fact, cutoff frequencies below $10 \mathrm{GHz}$ are possible in impedance transformers that are only $2 \mathrm{~mm}$ long).

\section{Slow-wave Impedance Transformer}

We have developed a new type of IT loaded with fins, ITF. The concept of this new type of IT has its roots in slowwave electrode theory ${ }^{8}$ where capacitive elements in the form of fins are added to coplanar strips. The addition of the capacitive elements to the coplanar electrodes significantly increases the capacitance per-unit-length while having negligible effect on the inductance of the line. As a result, increasing the capacitance of the CPW line not only lowers the characteristic impedance but also increases the effective microwave index, $n_{\text {eff }}$, i.e., adding extra capacitance decreases the propagation speed of the microwave. Therefore, instead of gradually tapering the center electrode to change the line impedance, one can gradually increase or decrease the fin capacitance to achieve the tapering effect.

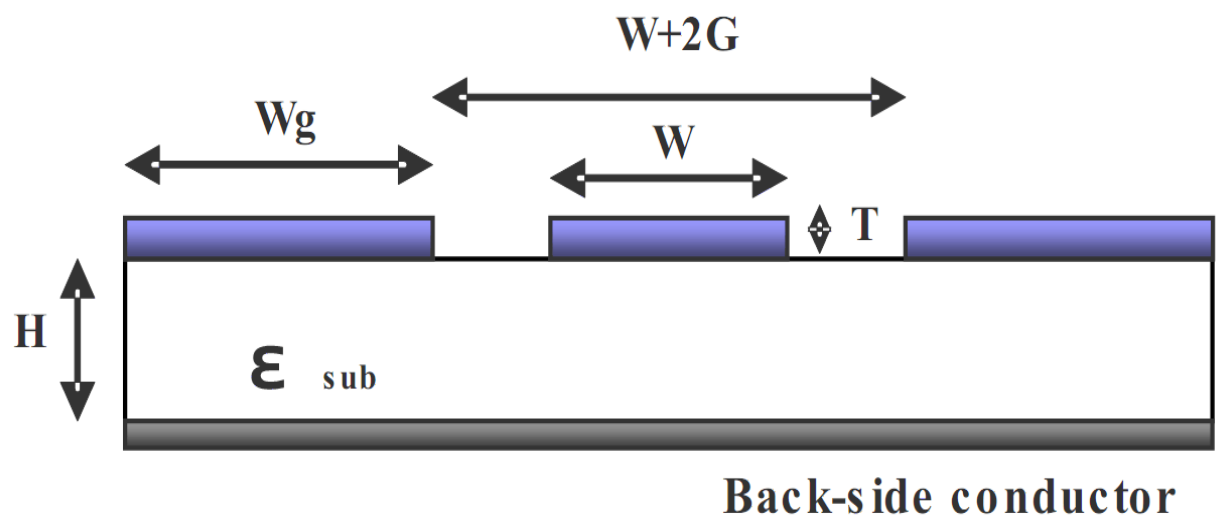

Figure 1. CPW layout cross-sectional view. 


\section{Low Impedance High Impedance}

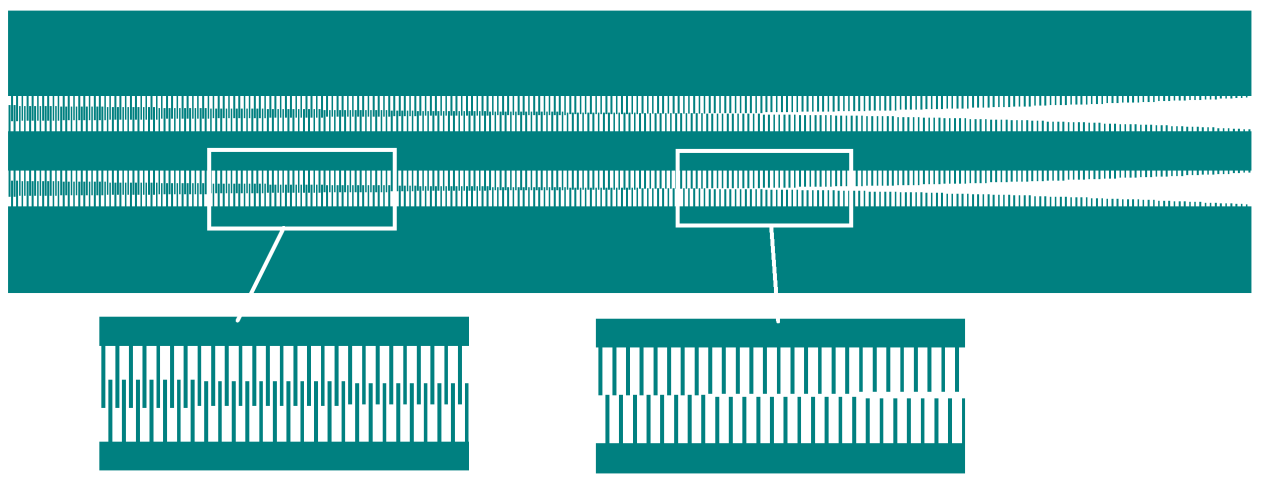

Figure 2. Typical ITF structure layout.

Geometry-wise, the ITF has a uniform width center electrode, which translates to low electrode loss, with fins gradually growing longer on both the center and the ground conductors along the length of the ITF. The low impedance end of the ITF has longer fins as compared to the high impedance end, see Figure 2.

The impedance value represented by a section of CPW loaded with interdigitated capacitive fins can be determined using SONNET ${ }^{\circledR}$ V.12 (this program can be used to compute the impedance of the line and $n_{\text {eff }}{ }^{9}$ ). Once a table of impedance values corresponding to different fin geometries is obtained, ITF can be designed by matching the impedance values along the length of the ETT to particular fin geometries. As expected, the effective microwave indices are higher at the low impedance end of the ITFs, where the capacitive loading of the fins is greatest. By employing the capacitive loading fins, $n_{\text {eff }}$ rises above 2.6 .

When ETT and ITF have the same cutoff frequency, the electrode length of the ITF is always shorter due to the increased values of $n_{\text {eff }}$ that are used. To demonstrate this, in Figure 3 the device characteristics of an ITF (ITF1) are compared with those of an exponentially tapered impedance transformer (ETT1). Both devices match $24 \Omega$ to $57 \Omega$ and $\mathrm{W}+2 \mathrm{G}=170 \mu \mathrm{m}$. ITF 1 employs $4 \mu \mathrm{m}$ wide fins and is $2 \mathrm{~mm}$ long. In contrast, ETT1 does not utilize any capacitive loading fins and is $2.75 \mathrm{~mm}$ long. To simulate electrode loss for both devices, the metal layer is chosen to be $0.5 \mu \mathrm{m}$ thick gold.

As shown in Figure 3, the cutoff frequencies of ITF1 and ETT1 are both $18.5 \mathrm{GHz}$. The S21 level of ITF1 shows a similar level of performance as that of ETT1. However, ETT1 is $37.5 \%$ longer than ITF1. By using ITF, one can achieve the same device performance with shorter electrode length, saving on-chip real-estate. When narrower fins are used, even greater real-estate savings can be achieved.

However, as the fins become longer and narrower, current crowding in the fins induces severe electrode loss above 40 GHz. This limits the upper $3 \mathrm{~dB}$ frequency of the ITF. In order to reduce electrode loss, one can use a combination of tapered center electrode and fin sections to design the impedance transformer, as explained in the following section.

\section{Impedance Matching Range of ITF}

Given the same minimum feature size of $1 \mu \mathrm{m}$ for fabrication, ITF can match impedances to lower values than ETT can. This is due to the excess amount of capacitance introduced by the interdigitated fins. In our simulations, we analyze ITFs with two different values of $\mathrm{W}+2 \mathrm{G}$, namely $170 \mu \mathrm{m}$ and $260 \mu \mathrm{m}$ and call these Type A and Type B devices, respectively (note: $\mathrm{Wg}=200 \mu \mathrm{m}$ for both types). Table 1 summaries the impedance matching ranges of ITF and of tapered CPW without fins. 

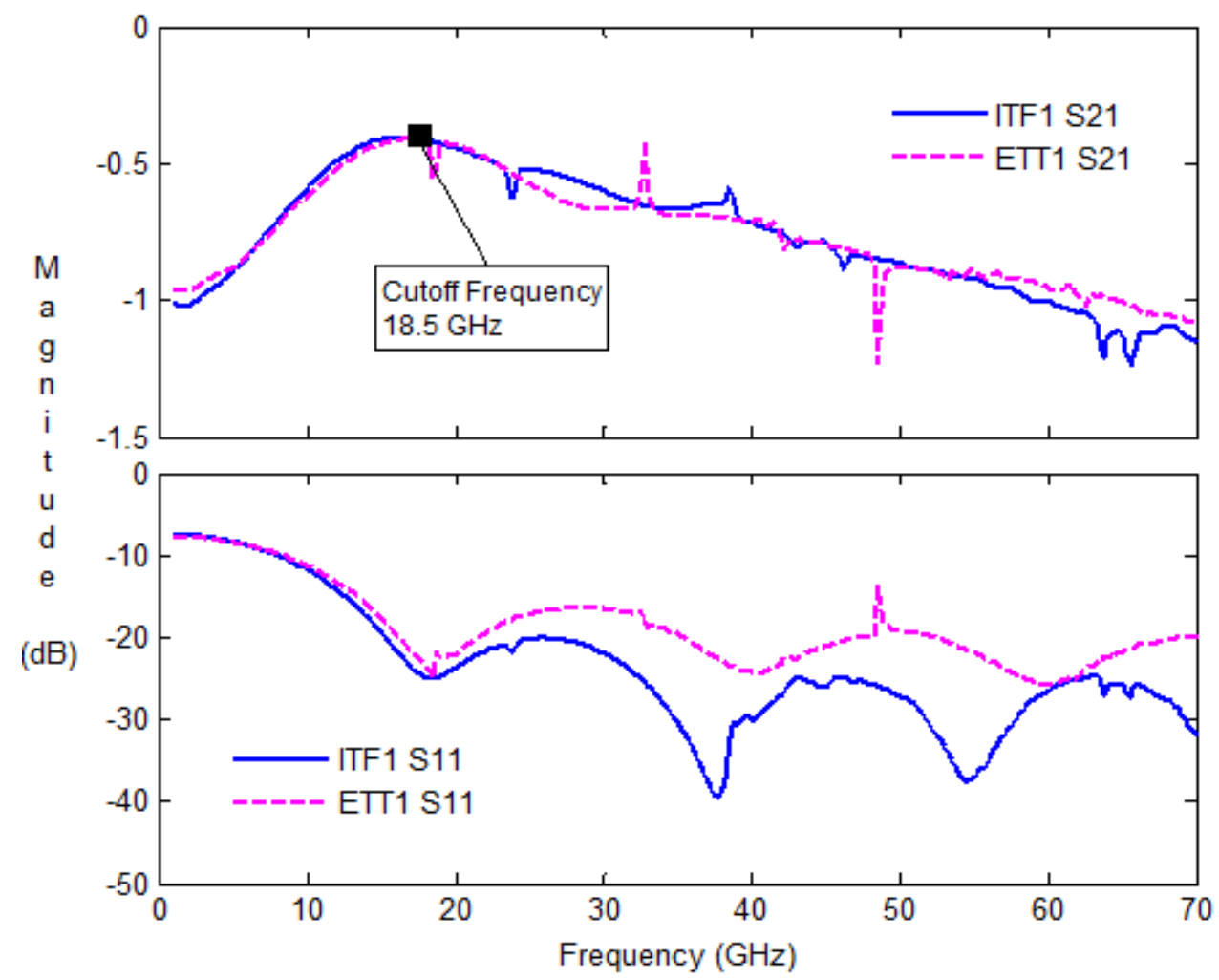

Figure 3. Device characteristics of ITF1 and ETT1.

\begin{tabular}{|l|c|c|c|}
\hline Electrode Type & Fin Width & Impedance Range $(\Omega)$ & $n_{\text {eff }}$ \\
\hline \multirow{2}{*}{$\begin{array}{l}\text { Type A: } \\
\mathrm{W}+2 \mathrm{G}=170 \mu \mathrm{m} \\
\mathrm{W}=60 \mu \mathrm{m} \\
\mathrm{G}=55 \mu \mathrm{m}\end{array}$} & $1 \mu \mathrm{m}$ & $12.5 \sim 57.8$ & $2.65 \sim 11.1$ \\
\cline { 2 - 4 } & $2 \mu \mathrm{m}$ & $17.2 \sim 57.8$ & $2.65 \sim 8.42$ \\
\cline { 2 - 4 } & $4 \mu \mathrm{m}$ & $22.5 \sim 57.8$ & $2.65 \sim 6.58$ \\
\cline { 2 - 4 } & Tapered CPW (no fins) & $23.9 \sim 193.8$ & 2.65 \\
\hline \multirow{2}{*}{$\begin{array}{l}\text { Type B: } \\
\mathrm{W}+2 \mathrm{G}=260 \mu \mathrm{m} \\
\mathrm{W}=60 \mu \mathrm{m} \\
\mathrm{G}=100 \mu \mathrm{m}\end{array}$} & $1 \mu \mathrm{m}$ & $10.1 \sim 68.5$ & $2.65 \sim 15.3$ \\
\cline { 2 - 4 } & $2 \mu \mathrm{m}$ & $14.1 \sim 68.5$ & $2.65 \sim 11.6$ \\
\cline { 2 - 4 } & $4 \mu \mathrm{m}$ & $19.0 \sim 68.5$ & $2.65 \sim 8.91$ \\
\cline { 2 - 4 } & Tapered CPW (no fins) & $24.5 \sim 229.1$ & 2.65 \\
\hline
\end{tabular}

Table 1. Impedance matching ranges of the impedance transformers for various fins widths.

The dimensions of $\mathrm{W}$ and $\mathrm{G}$ for each type of structure are listed in the first column of Table 1. In our ITF simulations, $\mathrm{W}$ for the fin sections is set to $60 \mu \mathrm{m}$ to minimize center electrode loss at high frequencies. $\mathrm{G}$ is chosen so that the fins have $54 \mu \mathrm{m}$ and $99 \mu \mathrm{m}$ space to grow in Type A and Type B devices, respectively. The impedance matching ranges of various widths of fins are shown in the third column. For example, in Type A devices, $1 \mu \mathrm{m}$ wide fins cover the impedance matching range from $12.6 \sim 57.8 \Omega$ when the length of the fins varies from $0 \mu \mathrm{m}$ to $54 \mu \mathrm{m}$. The 
corresponding values of $n_{\text {eff }}$ are shown in the fourth column. As shown in Table 1, the impedance matching range of various kinds of fin sections and regular CPW taper overlap, i.e., $30 \Omega$ can be designed by using $1 \mu \mathrm{m}$ wide fin sections, $2 \mu \mathrm{m}$ wide fin sections, $4 \mu \mathrm{m}$ wide fin sections, and tapered CPW without loading fins.

For a given center electrode width, adding capacitive loading fins to the CPW can only reduce the impedance of the line, but not increase it. Therefore, the highest impedance of the line can only be reached by narrowing the center electrode. In Type A structures, a conventional CPW taper is able to cover an impedance matching range from $\sim 23.9 \Omega$ to $\sim 193.8$ $\Omega$. By introducing tightly packed fins, the designer can extend the lower impedance matching range of the CPW taper. As the fins become longer and narrower, the added capacitance per unit length increases dramatically. Impedances as low as $\sim 12.5 \Omega$ can be reached with $1 \mu \mathrm{m}$ wide, $54 \mu \mathrm{m}$ long fins in Type A structures (with narrower and longer fins, lower impedance values can be reached). This makes matching to low impedance devices possible given the same onchip real-estate. Correspondingly, $n_{\text {eff }}$ reaches 11.1 for this fin geometry. In Type B structures, a possible impedance matching range from $10.1 \Omega$ to $\sim 229.1 \Omega$ can be achieved.

The overall attenuation of the impedance transformer is attributed to the cumulative loss of all ITF sections (here we use the term ITF to describe any impedance transformers employing capacitive fins). In order to minimize electrode loss due to the fins above $40 \mathrm{GHz}$, the designer can employ capacitive fins when matching impedance values that are below the lower impedance matching range of the CPW taper, e,g., in the range from $\sim 12.5 \Omega$ to $\sim 23.9 \Omega$ for Type A structures. Adopting this approach results in a combined capacitively-loaded/tapered structure such as that depicted in Figure 4.

A $2 \mathrm{~mm}$ long capacitively-loaded/tapered ITF structure matching $12.5 \Omega$ to $100.5 \Omega$ (ITF2), in which $\mathrm{W}+2 \mathrm{G}=170 \mu \mathrm{m}$, was studied. In order to minimize electrode loss at high frequencies, fin sections are only used below $24 \Omega$. In particular, $1 \mu \mathrm{m}$ wide and $2 \mu \mathrm{m}$ wide fins are used in combination to extend the lower impedance matching range. The center electrode is tapered to reach $100.5 \Omega$ at the high impedance end of the ITF. The device characteristics of ITF2 are compared with those of ETT2 (ETT2 is a $2.25 \mathrm{~mm}$ long exponentially tapered impedance transformer, matching $24 \Omega$ to $100.5 \Omega$ ). As shown in Figure 5, the cutoff frequency of each device is $21.25 \mathrm{GHz}$. The S21 level of ITF2 shows a similar level of performance as that of ETT2. However, ITF2 is $0.25 \mathrm{~mm}$ shorter than ETT2 and is able to match down to $12.5 \Omega$. Following this design approach, low-loss, ultra wideband impedance transformers capable of matching impedances from below $10 \Omega$ to over $200 \Omega$ should be realizable.

Low Impedance

High Impedance

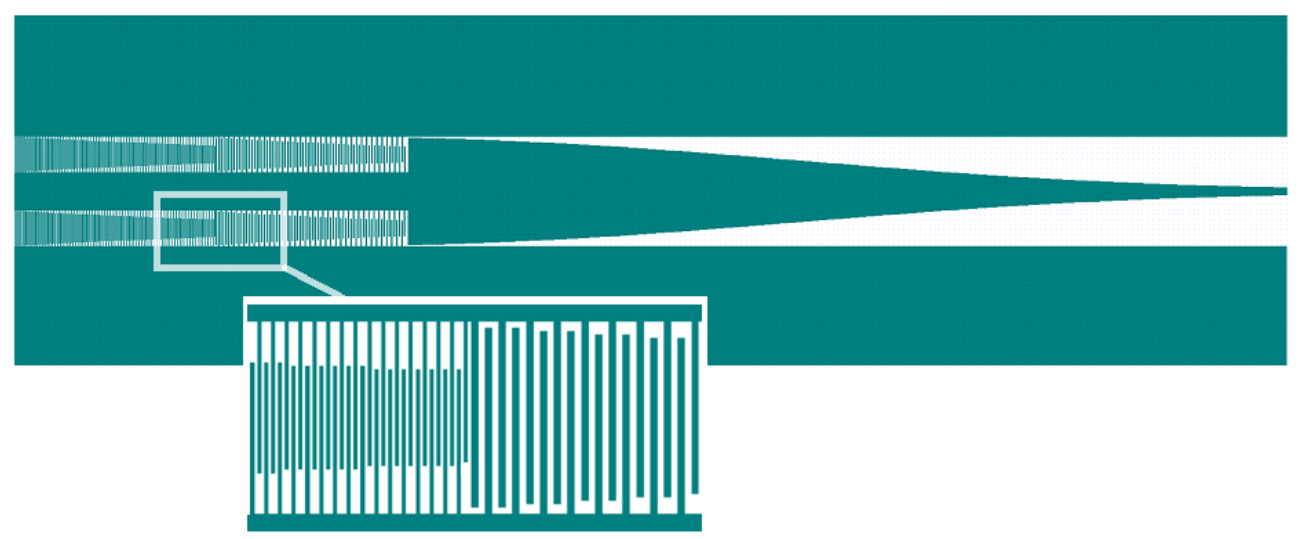

Figure 4. Schematic layout of ITF2. 


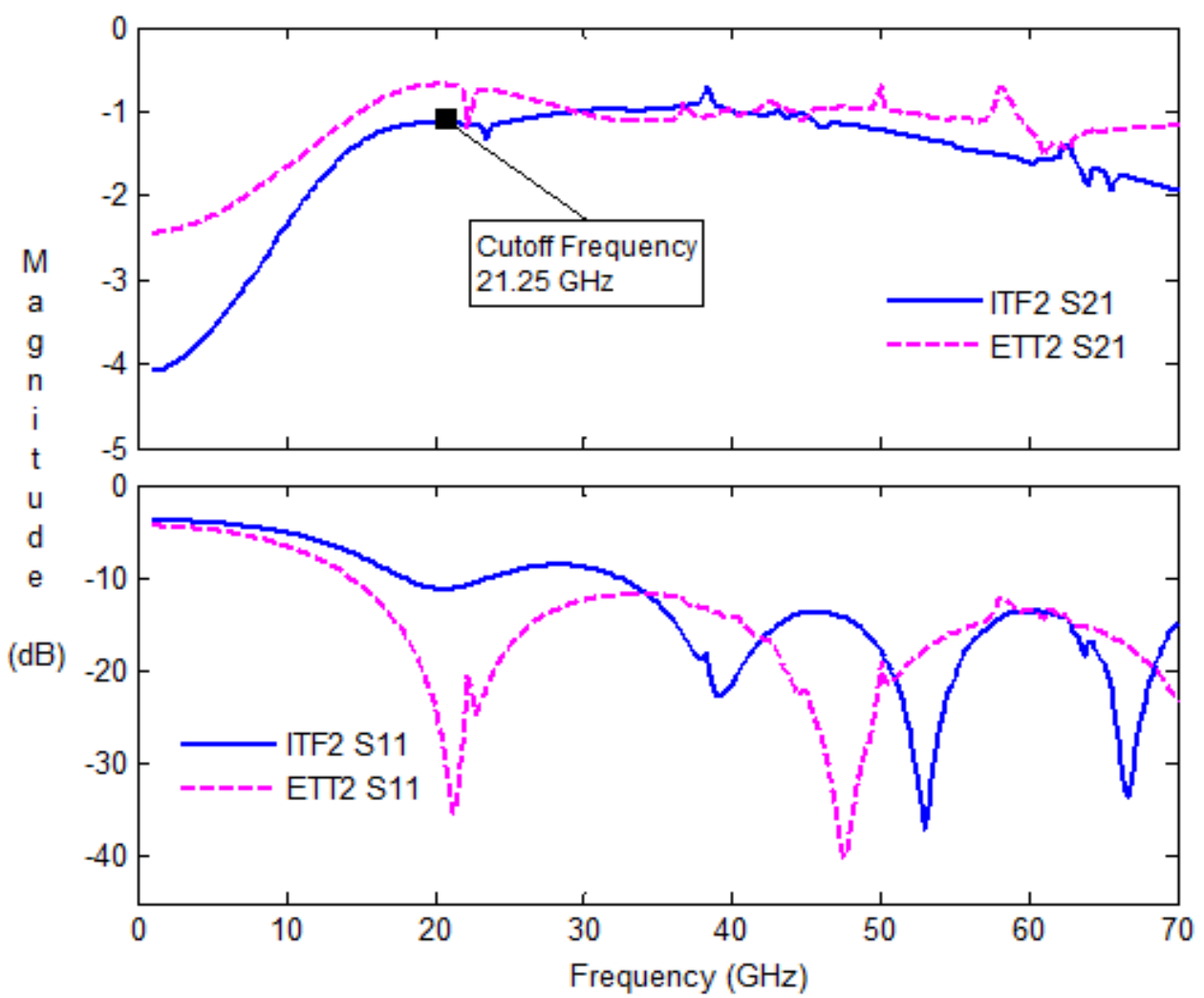

Figure 5. Device characteristics of ITF2 and ETT2.

\section{Conclusions}

Impedance transformers loaded with capacitive fins suitable for high frequency ultra wideband impedance matching are presented. We have demonstrated that ITFs are capable of matching $\sim 10 \Omega$ to $\sim 229 \Omega$ on $650 \mu \mathrm{m}$ thick GaAs substrates up to at least $70 \mathrm{GHz}$. Impedance transformers matching even lower impedance values are possible. The ITF design approach can be applied to other types of impedance transformer, such as triangularly tapered transformers and Klopfenstein tapered transformers, to extend their impedance matching ranges and to save on-chip real-estate.

\section{Acknowledgements}

The authors would like to thank Sonnet Software, Inc., for making SONNET ${ }^{\circledR}$ available to them at their educational rates. Also, this work was supported by grants from the Natural Sciences and Engineering Research Council of Canada which the authors gratefully acknowledge.

\section{References}

[1] Jensen, T., Zhurbenko, V., Krozer, V., and Meincke, P.,"Coupled Transmission Lines as Impedance Transformer", IEEE Trans. Microw. Theory Tech., 55(12), (2007).

[2] Ang, K. S., Lee, C. H., and Leong, Y. C., "A Broad-Band Quarter-Wavelength Impedance Transformer With Three Reflection Zeros Within Passband", IEEE Trans. Microw. Theory Tech., 52(12), (2004).

[3] Nesic, A.; Jovanovic, S., "A new small size wideband impedance transformer", Telecommunications in Modern Satellite, Cable and Broadcasting Services, 7th International Conference, (2005) 
[4] Kanaya, H., Nakamura, T., Kawakami, K., and Yoshida, K., "Design of Coplanar Waveguide Matching Circuit for RF-CMOS Front-End", Electronics and Communications in Japan, Part 2, Vol. 88, No. 7, 2005.

[5] Kanaya, H., Pokharel, R.K., Koga, F., Arima, Z., Kim, S., Yoshida, K., "Design of coplanar waveguide on-chip impedance-matching circuit for wireless receiver front-end", RFICS, (2006).

[6] Misra, D. K., [Radio-Frequency and Microwave Communication Circuits: Analysis and Design, 2nd Edition], Chapter 7(2004).

[7] Riaziat, M., Majidi-Ahy, R., and Feng, I-J., "Propagation Modes and Dispersion Characteristics of Coplanar Waveguides", IEEE Trans. Microw. Theory Tech., 38(3), (1990).

[8] Jaeger, N. A. F., and Lee, Z. K. F., "Slow-wave electrode for use in compound semiconductor electrooptic modulators," IEEE J. Quantum Electron., 28, 1778-1784 (1990).

[9] Spickermann, R., and Dagli, N., "Experimental Analysis of Millimeter Wave Coplanar Waveguide Slow Wave Structures on GaAs", IEEE Trans. Microw. Theory Tech., 42(10), (1994). 